\title{
Article \\ Chirality, Gelation Ability and Crystal Structure: Together or Apart? Alkyl Phenyl Ethers of Glycerol as Simple LMWGs
}

\author{
Alexander A. Bredikhin * \\ Olga A. Lodochnikova (D) \\ Aidar T. Gubaidullin, Zemfira A. Bredikhina (D, Robert R. Fayzullin ${ }^{D}$ and
}

FRC Kazan Scientific Center of RAS, Arbuzov Institute of Organic and Physical Chemistry, Arbuzov St., 8, 420088 Kazan, Russia; aidar@iopc.ru (A.T.G.); zemfira@iopc.ru (Z.A.B.); fayzullin@iopc.ru (R.R.F.); olga@iopc.ru (O.A.L.)

* Correspondence: baa@iopc.ru

Citation: Bredikhin, A.A.;

Gubaidullin, A.T.; Bredikhina, Z.A.; Fayzullin, R.R.; Lodochnikova, O.A. Chirality, Gelation Ability and Crystal Structure: Together or Apart? Alkyl Phenyl Ethers of Glycerol as Simple LMWGs. Symmetry 2021, 13, 732. https://doi.org/10.3390/sym13040732

Academic Editor: Angela Patti

Received: 24 March 2021

Accepted: 16 April 2021

Published: 20 April 2021

Publisher's Note: MDPI stays neutral with regard to jurisdictional claims in published maps and institutional affiliations.

Copyright: (c) 2021 by the authors. Licensee MDPI, Basel, Switzerland. This article is an open access article distributed under the terms and conditions of the Creative Commons Attribution (CC BY) license (https:// creativecommons.org/licenses/by/ $4.0 /)$.

\begin{abstract}
Chiral recognition plays an important role in the self-assembly of soft materials, in particular supramolecular organogels formed by low molecular weight gelators (LMWGs). Out of 14 pairs of the studied racemic and enantiopure samples of alkyl-substituted phenyl ethers of glycerol, only eight enantiopure diols form the stable gels in nonane. The formation of gels from solutions was studied by polarimetry, and their degradation with the formation of xerogels was studied by the PXRD method. The revealed crystalline characteristics of all studied xerogels corresponded to those for crystalline samples of the parent gelators. In addition to those previously investigated, crystalline samples of enantiopure para-n-alkylphenyl glycerol ethers [alkyl = pentyl (5), hexyl (6), heptyl (7), octyl (8), nonyl (9)] and racemic 3-(3,5-dimethylphenoxy)propane-1,2-diol (rac-14) have been examined by single crystal X-ray diffraction analysis. Among 22 samples of compounds 1-14 studied by SC-XRD, seven different types of supramolecular motifs are identified, of which only two are realized in crystals of supramolecular gelators. An attempt was made to relate the ability to gel formation with the characteristics of the supramolecular motif of a potential gelling agent, and the frequency of formation of the motif, required for gelation, with the chiral characteristics of the sample.
\end{abstract}

Keywords: chirality; aryl glycerol ethers; supramolecular organogel; optical rotation; powder X-ray diffraction (PXRD); single crystal X-ray diffraction (SC-XRD); crystal packing

\section{Introduction}

Supramolecular hydro- and organogels, which owe their existence to relatively small molecules (called low-molecular-weight gelators, LMWG), are classical nanoobjects [1]. Supramolecular gels (SMG) moved into the category of the scientific mainstream in the last decade of the 20th century, when the number of annual publications on this topic increased abruptly and exceeded the limit of several thousand [2]. Since then, this number has only increased. So, over the past 10 years, several monographs have been published on this topic [3-6]. Many reviews highlight the current state of the subject $[2,7,8]$, instrumental methods for the study of SMG $[9,10]$, the structure of supramolecular associates arising in the gelling process [11,12], the relationship between gelation and the solvent's nature [13], progress in the design of new LMWG [14-16], and the realized and potential use of SMG in practice [17-19]. However, according to the eminent Richard G. Weiss, despite the abundance of information, "There will be very limited advancement in understanding two fundamental aspects of molecular gels: (1) Why, when, and how do they form? and (2) How can their properties be predicted a priori?" [20]. The main reason for this state of affairs is the frighteningly large number of factors that determine the very possibility of the existence of supramolecular gels, the differences in their morphology and properties. In turn, this creates a situation in which "the differences among some gel types are greater than their similarities" [21]. 
At the very beginning of the "gel boom", a feature inherent in chiral compounds acting as gelators was noticed. While many of them form gels (not always identical in characteristics) regardless of the enantiomeric composition, relatively simple compounds of this class exhibited their specific properties only in the enantiopure form and did not exhibit in the racemic form, and vice versa. Examples of such behavior are given in reviews [22,23] and this effect has also been noted in recent publications [24-27]. Subsequently, this minor mystery of chiral gels was obscured by other, significant in meaning and spectacular in illustrations, problems of supramolecular chirality [28,29] and chiral recognition [30], which are clearly manifested in nanostructured media. However, being unresolved, the problem of the dependence of gelation on the enantiomeric state of the gelator does not allow it to be written off to the archive.

To interpret this effect, the general concept of crystallization of a chiral substance was used, according to which racemates crystallize more easily than enantiopure samples [23], and the so-called "Fuhrhop's chiral bilayer effect" [31,32]. According to the latter, the hydrophobic effect impresses sheet-like bilayer structures and produces the water-insoluble amphiphile molecules aggregates of cylindrical shape. It is believed that the reverse process, the transformation of cylindrical fibrils into bilayers and their subsequent laying into a crystal lattice, is easier for heterochiral (racemic) associates, which are thus removed from the liquid medium. On the contrary, homochiral (enantiopure) fibrils should have difficulty in the formation of bilayer crystalline nuclei. For this reason, they accumulate in the liquid medium and give rise to gels.

It should be noted right away that all such considerations are too general; moreover, in reality, the energies of homo- and heterochiral crystal packings differ not so significantly, and these differences are not always in favor of the latter. Further, hydrophobic effects are significant for hydrogels, whereas the effect of chirality-dependent gelation is characteristic of organogels as well. Finally, in most attempts to connect the features of gelation with the features of gelator crystallization, real information about the crystalline state of the latter is either absent or is based on the study of models [33].

It is clear that, given the variety of SMGs, it is not easy, if possible at all, to identify the universal reason for the chiral-dependent gelation phenomenon, but, in our opinion, one can count on particular solutions if the problem statement itself is simplified and made more rigorous. At the first stage, the influence of the medium on the gelation process should be minimized. Obviously, hydrocarbons are the best solvents for this purpose. Further, it is necessary to focus on gelling agents of the same type and limit the consideration to single-component organogelators, excluding salts and coordination compounds from the analysis. Finally, the structure of the gelator itself should be simplified as much as possible, i.e., the set of functional groups present in it should be minimized.

All these conditions are met by the para-tolyl ether of glycerol 1, which is apparently the simplest possible chiral LMWG, which forms stable gels in alkanes in an enantiopure form and is incapable of supramolecular gelation in the form of a racemate [34]. In this work, in search of causal relationships between the nature of chiral-dependent gelation and the crystal structure of the gelling agent, we propose to compare the previously known and newly obtained data on the properties of ether $\mathbf{1}$ and the representative family of structurally related alkyl-substituted phenyl ethers of glycerol 2-14 (Scheme 1). 
<smiles>CCc1ccc(OC[C@@H](O)CO)cc1</smiles>

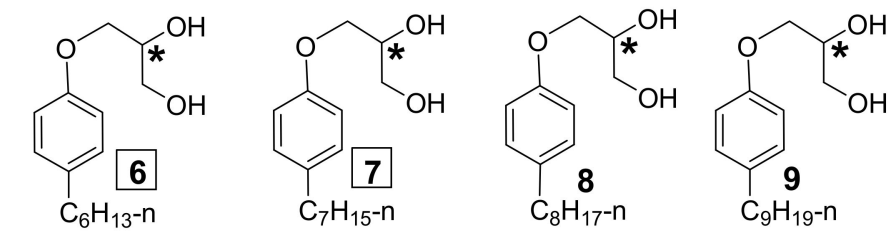

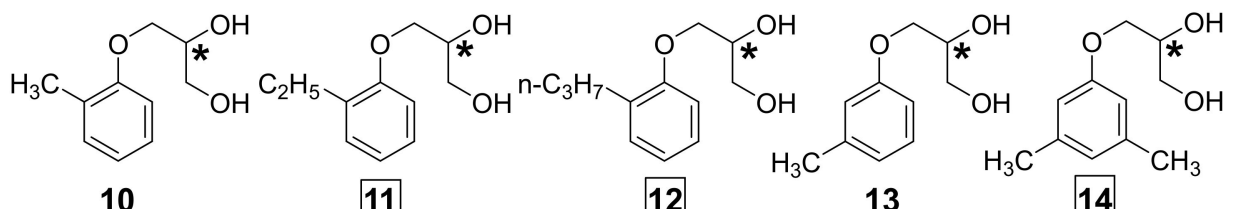

Scheme 1. Alkylphenyl ethers of glycerol 1-14 investigated in the paper. The chiral center is marked with an asterisk; the boxes indicate the numbers of compounds showing a tendency to supramolecular gelation.

\section{Materials and Methods}

\subsection{Instrumentation}

Melting points for general purposes were determined using a Boëtius apparatus and are uncorrected. Optical rotations were measured on a Perkin-Elmer model 341 polarimeter (concentration $\mathrm{c}$ is given as $\mathrm{g} / 100 \mathrm{~mL}$ ). Enantiomeric purity (ee) was checked by HPLC analysis performed on a Shimadzu LC-20AD system controller, and a UV monitor $275 \mathrm{~nm}$ was used as a detector. The columns used, from Daicel Inc., were Chiralcel OD or OD-H $(0.46 \times 25 \mathrm{~cm})$; eluent hexane: isopropanol $=4: 1 \mathrm{v} / \mathrm{v}$; flow rate: $1 \mathrm{~mL} \cdot \mathrm{min}^{-1}$.

\subsection{Materials}

The syntheses and characterization of racemic and enantiopure methyl substituted glycerol ethers 1, 10, 13 were described by us earlier [34,35]; rac- and (S)-3,5-dimethyl substituted ethers $\mathbf{1 4}$ were described in our work [36]; ortho-substituted ethers $\mathbf{1 1}$ and 12 were described in our work [37]. The synthesis of the racemic and (S)-para-n-alkylphenyl glycerol ethers 2-9 has been described in detail in our previous work [38]. Enantiopure diols $(R)$-2-9 and $(R)-14$ were obtained from $(R)$-3-chloropropane-1,2-diol (98\% ee) and the appropriately substituted phenol by analogy to the published procedures for the racemic samples [38]. The single crystals of the compounds (R)-2-4 were investigated by X-ray diffraction by us earlier [39]. The single crystals of the compounds $(R)-5-9$ and rac-14, first investigated by $\mathrm{X}$-ray diffraction in this paper, were prepared by slow evaporation of solutions of the corresponding samples in a mixture of n-hexane and ethyl acetate $(8: 2)$. Various methods were used to obtain crystals of the enantiopure compound 14. When growing crystals of (R)-14 by slow evaporation of its solutions in hexane, $\mathrm{CCl}_{4}$, or in an EtOAc-hexane mixture, as well as by layering in hexane/methylene chloride or cyclohexane/methylene chloride systems, very thin intertwined threads were formed (Figure 1b). This made it impossible to study this sample by SC-XRD.

Characteristics of the obtained crystals agree with literature data and are shown below: (R)-3-(4-n-Pentylphenoxy)-propane-1,2-diol, (R)-5: $\operatorname{mp} 52{ }^{\circ} \mathrm{C}, \mathrm{cp} 79{ }^{\circ} \mathrm{C},[\alpha]_{\mathrm{D}}{ }^{20}-7.6(c 1$, $\mathrm{EtOH}) ;$ ee $99.6 \%$.

(R)-3-(4-n-Hexylphenoxy)propane-1,2-diol, (R)-6: mp $48{ }^{\circ} \mathrm{C}, \mathrm{cp} 84{ }^{\circ} \mathrm{C},[\alpha]_{\mathrm{D}}{ }^{20}-6.4(c 1$, EtOH); eе $99.6 \%$.

(R)-3-(4-n-Heptylphenoxy)propane-1,2-diol, (R)-7: mp $51{ }^{\circ} \mathrm{C}, \mathrm{cp} 86{ }^{\circ} \mathrm{C},[\alpha]_{\mathrm{D}}{ }^{20}-6.5(c)$, $\mathrm{EtOH}) ;$ eе $98 \%$ 
(R)-3-(4-n-Octylphenoxy)propane-1,2-diol, (R)-8: $\mathrm{mp} 60{ }^{\circ} \mathrm{C}, \mathrm{cp} 88^{\circ} \mathrm{C},[\alpha]_{\mathrm{D}}{ }^{20}-5.8(c$, EtOH), ee $99.7 \%$.

(R)-3-(4-n-Nonylphenoxy)propane-1,2-diol, (R)-9: careful reinvestigation of the diol scal-9 enabled us to reveal that the true melting and clearing points (cp) are $56{ }^{\circ} \mathrm{C}$ and $89{ }^{\circ} \mathrm{C}$ respectively; $[\alpha]_{\mathrm{D}}^{20}=-5.6(c 1, \mathrm{EtOH})$, ee $99.3 \%$.

rac-3-(3,5-Dimethylphenoxy)propane-1,2-diol, rac-14: $\mathrm{mp} 63.5-65{ }^{\circ} \mathrm{C}$.

(R)-3-(3,5-Dimethylphenoxy)propane-1,2-diol, (R)-14: mp 73-74 ${ }^{\circ} \mathrm{C},[\alpha]_{\mathrm{D}}{ }^{20}-7.8(c$ 1.0, $\mathrm{EtOH})$, ee $99.9 \%$ \{cf. lit. [36] for (S)-14: $\left.\mathrm{mp} 72-73{ }^{\circ} \mathrm{C},[\alpha]_{\mathrm{D}}{ }^{20}=+7.3(c 1.2, \mathrm{EtOH})\right\}$.
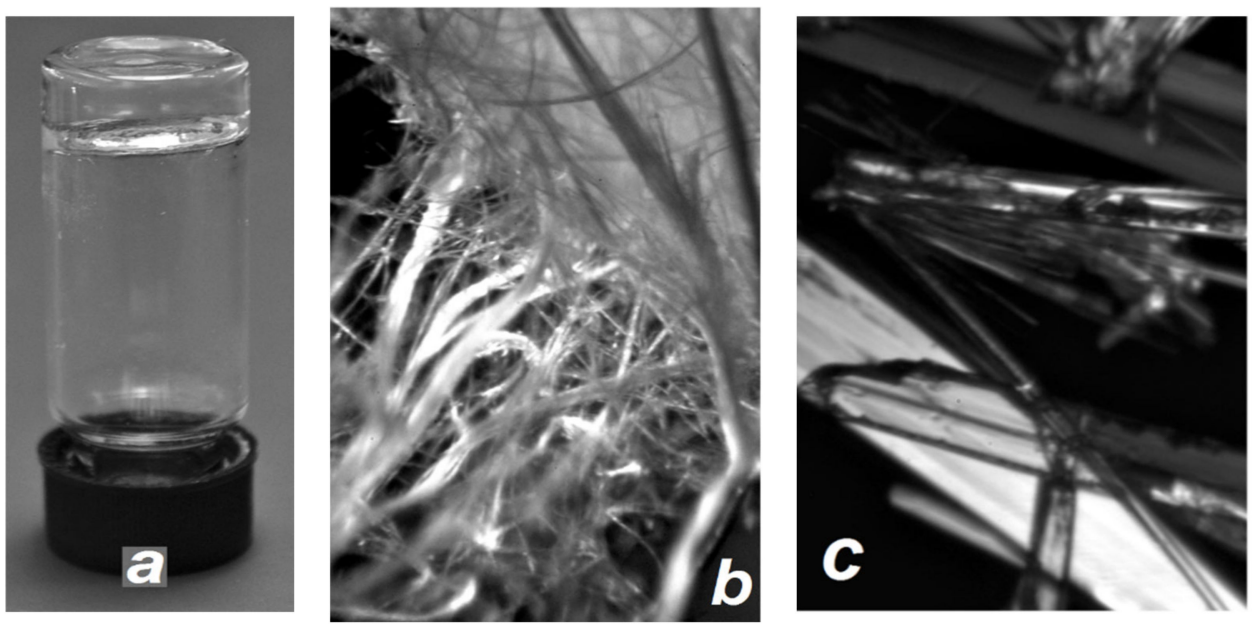

Figure 1. (a) Gel formed by (R)-14 and n-nonane; $(R)-\mathbf{1 4}$ (b) and rac-14 (c) crystals; (microscope magnification $\sim 16 \times$ ).

\subsection{Gelation Test}

The corresponding diol 1-14 $(1 \div 4 \mathrm{mg})$ and nonane $(\sim 1 \mathrm{~mL})$ was put in a $2 \mathrm{~mL}$ vial and heated until the solid was dissolved. The resulting solution was cooled in air to room temperature, and then left for $30 \mathrm{~min}$ at this temperature. The state of the materials was evaluated by the "stable-to-inversion of a vial" method. The stable gels were observed only for scal-diols 1, 4-7, 11, 12, 14. Then the excess solvent was poured off. The gel was weighed and the minimum gelator concentration (MGC) necessary for gelation of nonane at $25{ }^{\circ} \mathrm{C}\left[\left(\mathrm{m}_{\text {gelator }} / \mathrm{m}_{\text {gel }}\right) 100 \%\right]$ was determined (Table 1$)$. For all the identified gelators, MGC values were in the range of $0.1-0.5 \mathrm{~W} \%$.

\subsection{Optical Rotations Measurements of Gels}

All the solutions were put into a glass tube by known amounts of a sample [Shimadzu AUW 120D analytic balance (accuracy $\pm 0.01 \mathrm{mg}$ )] and nonane, and the weight fraction of the mixture was determined. The range of the investigated concentrations of solutions of enantiopure diols 1,11 and 14 was $0.7-2.2 \mathrm{mg} \mathrm{mL}^{-1}$, that is, $\sim 4-11 \mathrm{mmol} \mathrm{L}^{-1}$. After the mixtures were heated up to $50{ }^{\circ} \mathrm{C}$, the homogeneous solutions were poured into a Perkin-Elmer thermostated glass cell (pathlength $100 \mathrm{~mm}$, volume $1.0 \mathrm{~mL}$ ) and placed in a polarimeter. The cell was cooled at a rate of about one degree per minute to a temperature of $20^{\circ} \mathrm{C}$, while recording the values of specific rotation over time. The exact values of the concentration of the stock solutions and the specific rotations at different wavelengths of the gels, obtained from them, are given in Table S1.

\subsection{Single Crystal X-ray Analysis}

X-ray diffraction study of the single crystals $(R)-5-(R)-\mathbf{9}$, and rac-14 was performed on a Bruker SMART APEX II CCD automated three-circle diffractometer at a temperature of 293(2) K [296(2) K for rac-14]: graphite monochromator, $\lambda$ (Mo K $\alpha$ ) $=0.71073 \AA$, $\omega$-scan mode with a step of $0.5^{\circ}$. Single crystals of appropriate sizes were placed on glass fibers at a random orientation. Preliminary unit cell parameters were determined using three runs 
(different positions with respect to angle $\varphi$ ) with 12 frames in each run ( $\omega$ scanning). Data collection and indexing, determination, and refinement of unit cell parameters were carried out using the APEX3 (v2019.11-0, Bruker AXS) software package. Numerical absorption correction based on the crystal shape, additional spherical absorption correction, and systematic error correction were performed using the SADABS-2016/2 software. The structures were solved by direct methods using the SHELXT-2018/2 program [40] and refined by full-matrix least-squares on $F^{2}$ using the SHELXL-2018/3 program [41]. Nonhydrogen atoms were refined anisotropically. Positions of $\mathrm{H}[\mathrm{O}]$ hydrogen atoms were determined from difference electron density maps and refined isotropically, except for $(R)-6$. The hydrogen atoms of methyl groups were calculated geometrically and refined using the rotation of the group with idealized bond angles. The remaining hydrogen atoms were refined using a riding model. The disorder, if present, was resolved using free variables and reasonable restraints on geometry and anisotropic displacement parameters. Most calculations were performed using the WinGX-2020.2 software package [42]. A crystallographic summary is given in Tables S2-S4. Mercury program packages [43] was used for figures preparation.

Deposition numbers 1,406,279 [(R)-5], 1,406,280 [(R)-6], 1,406,281 [(R)-7], 1,406,282 [(R)-8], 2,064,414 [(R)-9], and 2,071,436 (rac-14) contain the supplementary crystallographic data for this paper. These data are provided free of charge by the joint Cambridge Crystallographic Data Centre and Fachinformationszentrum Karlsruhe Access Structures service www.ccdc.cam.ac.uk/structures (accessed on 23 March 2021).

\subsection{Powder X-ray Analysis}

For the study, samples of gels in nonane were prepared, as well as samples of the initial powdery racemic and enantiopure compounds. The gels had the following sample concentrations: $(R)-435.6 \mathrm{mmol} \mathrm{L}^{-1}\left(7.9 \mathrm{mg} \mathrm{mL}^{-1}\right)$; $(R)-537.3 \mathrm{mmol} \mathrm{L}^{-1}\left(8.8 \mathrm{mg} \mathrm{mL}^{-1}\right)$; $(R)$ $637.2 \mathrm{mmol} \mathrm{L}^{-1}\left(9.3 \mathrm{mg} \mathrm{mL}^{-1}\right) ;(R)-730.0 \mathrm{mmol} \mathrm{L}^{-1}\left(8.0 \mathrm{mg} \mathrm{mL}^{-1}\right) ;(R)-1113.8 \mathrm{mmol} \mathrm{L}^{-1}$ (2.7 mg mL $\left.\mathrm{mg}^{-1}\right)$; $(R)-\mathbf{1 2} 13.3 \mathrm{mmol} \mathrm{L}^{-1}\left(2.8 \mathrm{mg} \mathrm{mL}^{-1}\right)$; $(R)-\mathbf{1 4} 8.15 \mathrm{mmol} \mathrm{L}^{-1}\left(1.6 \mathrm{mg} \mathrm{mL}^{-1}\right)$.

PXRD measurements were performed on an automatic Bruker D8 Advance diffractometer equipped with a Vario attachment and a Vantec linear position-sensitive detector using $\mathrm{Cu}$ radiation $(40 \mathrm{kV}, 40 \mathrm{~mA})$ monochromated by a curved Johansson monochromator $(\lambda \mathrm{CuK} \alpha 11.5406 \AA)$. Room-temperature data were collected in the reflection mode with a flat-plate sample. The samples in gel form were deposited on the surface of a silicon plate with minimal background scattering. Time-resolved powder diffraction patterns were recorded in the $2 \theta$ range between $1.5^{\circ}$ and $50^{\circ}$ in $0.008^{\circ}$ steps with a step time of $0.1 \mathrm{~s}$, the recording time for one scan was $10 \mathrm{~min}$. Powdered samples were also applied without pressure to the surface of a silicon plate, diffraction patterns were obtained in the $2 \theta$ range from 1.5 to $70^{\circ}$, with a step of $0.008^{\circ}$ and a set time of $0.1-1 \mathrm{~s}$. The samples were spun (15 rpm) throughout the data collection. Processing of the obtained data was performed using EVA software packages [44].

\section{Results and Discussion}

\subsection{Gelation Abilities of Compounds 1-14 and Some Gel Characteristics and Properties}

Preliminary information on the ability of some compounds presented in Scheme 1 to supramolecular gelation in various solvents was reported previously $[34,35,38,45]$. In this work, for the convenience of comparing the results, we investigated the possibility of gelation of the entire group of glycerol alkylphenyl ethers 1-14 in racemic and enantiopure forms in uniformly performed experiments using the nonane hydrocarbon as a liquid medium. The results are shown in Table 1.

From Table 1, it follows that of the 28 tested samples, eight showed a pronounced tendency to gel formation. If one evaluates gelation efficiency, for example, as the number of moles of nonane solvent retained by one mole of gelling agent, then the most effective is enantiopure para-tolyl ether of glycerol scal-1 with a molecular weight of 182 Daltons, one mole of which traps in the gel phase about 1400 moles of solvent. The second most effective was the 3,5-dimethylsubstituted phenyl glycerol ether scal-14, the gelling properties of 
which were for the first time described in this work (Figure 1a). One mole of this gelling agent is capable of holding up to 1100 moles of nonane. At the opposite end of the scale is the scal-4 gelator, which generates a turbid unstable gel prone to crystallization. More stable, but also turbid, gels are formed by enantiopure gelators 5-7 and 12. Completely transparent gels were formed by enantiopure samples of compounds 1, 11, 14 .

Table 1. Characteristics of the ability to supramolecular gelation in nonane, and the features of crystallization of racemic and enantiomeric samples of alkylphenyl ethers of glycerol 1-14.

\begin{tabular}{|c|c|c|c|c|c|}
\hline No & Sample & MGC $^{1}, w^{2} \%$ & Nonane/Gelator (mol/mol) & Group; Z,Z' & Motif $^{2}$ \\
\hline \multirow[b]{2}{*}{1} & $\mathrm{rac}$ & -3 & & Pc $(7) ; 4,2 .{ }^{4,5}$ & BL-3 \\
\hline & scal & 0.10 & 1420 & $P 2_{1} 2_{1} 2_{1}(\underline{19}) ; 4,1.4,5$ & BL-1 \\
\hline \multirow{2}{*}{2} & rac & - & & $P 2_{1} / \mathrm{c}(\underline{14}) ; 16,4 .^{6}$ & BL-4 \\
\hline & scal & - & & $P 2_{1} 2_{1} 2_{1}(\underline{19}) ; 4,1 .^{6}$ & BL-1 \\
\hline 3 & $\begin{array}{l}\text { rac } \\
\text { scal }\end{array}$ & - & & $P 2_{1}(\underline{4}) ; 2,1 .^{6}$ & BL-1 \\
\hline \multirow[b]{2}{*}{4} & rac & - & & $P 2_{1} / c(14) ; 4,1 .^{6}$ & BL-1 \\
\hline & scal & 0.55 & 313 & $P 2{ }_{1} 2_{1} 2_{1}(\underline{19}) ; 4,1 .^{6}$ & BL-1 \\
\hline \multirow{2}{*}{5} & rac & - & & & \\
\hline & scal & 0.26 & 792 & $P 2{ }_{1}{ }_{1} 2_{1}(\underline{19}) ; 4,1 .^{-7}$ & BL-1 \\
\hline \multirow[b]{2}{*}{6} & rac & - & & & \\
\hline & scal & 0.48 & 414 & $I 2(\underline{5}) ; 4,2 .^{7}$ & BL-2 \\
\hline \multirow[b]{2}{*}{7} & rac & - & & & \\
\hline & scal & 0.25 & 822 & $P 2_{1} 2_{1} 2_{1}(\underline{19}) ; 4,1 .^{7}$ & BL-1 \\
\hline \multirow{2}{*}{8} & rac & - & & & \\
\hline & scal & - & & $P 2_{1}(\underline{4}) ; 4,2.7$ & BL-2 \\
\hline \multirow[b]{2}{*}{9} & rac & - & & & \\
\hline & scal & - & & $P 2_{1}(\underline{4}) ; 4,2^{-7}$ & CL-1 \\
\hline 10 & $\begin{array}{l}\text { rac } \\
\text { scal }\end{array}$ & $\begin{array}{l}- \\
-\end{array}$ & & $P 2_{1}(\underline{4}) ; 4,2 .{ }^{5,8}$ & CL-2 \\
\hline \multirow{2}{*}{11} & rac & - & & $P 2{ }_{1}{ }_{1}{ }_{1}(19) ; 4,1 .^{9}$ & BL-1 \\
\hline & scal & 0.30 & 777 & & \\
\hline \multirow{2}{*}{12} & rac & - & & $P 2_{1} / n(\underline{14}) ; 4,1 .^{9}$ & BL-1 \\
\hline & scal & 0.39 & 776 & $P 2_{1}(\underline{4}) ; 4,2 .{ }^{9}$ & BL-1 \\
\hline \multirow{2}{*}{13} & $\mathrm{rac}$ & - & & $\mathrm{Pca}_{1}(\underline{29}) ; 8,2 .^{5}$ & BL-3 \\
\hline & scal & - & & $P 2_{1}(\underline{4}) ; 4,2^{5,8}$ & CL-2 \\
\hline \multirow{2}{*}{14} & rac & - & & $P 1(\underline{2}) ; 4,2 .{ }^{7}$ & $\mathrm{RR}$ \\
\hline & scal & 0.14 & 1090 & & \\
\hline
\end{tabular}

${ }^{1}$ Minimum gelator concentration. ${ }^{2} \mathrm{BL}-2 \mathrm{D}$ bilayer, CL-1D column, RR-1D ribbon of rings; see text for other details. ${ }^{3}$ Denotes no gelation; solution or precipitate formed. ${ }^{4}$ Ref. [34]. ${ }^{5}$ Ref. [35]. ${ }^{6}$ Ref. [39]. ${ }^{7}$ This work. ${ }^{8}$ Ref. [46]. ${ }^{9}$ Ref. [45].

To assess the thermal stability of supramolecular gels, different methods can be used $[9,10]$, the simplest of which is the steel ball test, according to which all gels studied in this work are destroyed in a narrow range of $38-40{ }^{\circ} \mathrm{C}$, depending on the structure and enantiomeric purity of the gelator. It is somewhat more difficult to determine the temperature range in which the formation of the gel phase begins and progresses intensively. It is well known that chiral gelators form chiral supramolecular nanostructures during gelation [11], which, in particular, affects the chiroptic characteristics of the gel. As a rule, such changes are recorded using circular dichroism spectra, and a similar experiment for the (S)-1 gel was described by us earlier [34].

The above-noted stability and transparency of hydrocarbon gels formed by enantiopure gelators 1, 11, and $\mathbf{1 4}$ make it possible to use polarimetry to study the formation of corresponding chiral gels. Figure 2 shows changes in specific rotation at a wavelength of $589 \mathrm{~nm}$ for solutions of these compounds initially heated to $50{ }^{\circ} \mathrm{C}$ during cooling to $20{ }^{\circ} \mathrm{C}$ at a rate of $1 \mathrm{deg}$ per minute. Table S1 shows the specific rotation values for the same supramolecular gels $30 \mathrm{~min}$ after the start of the cooling of the solution at different wavelengths. The sign of the optical rotation depends on the configuration of the original gelator. 


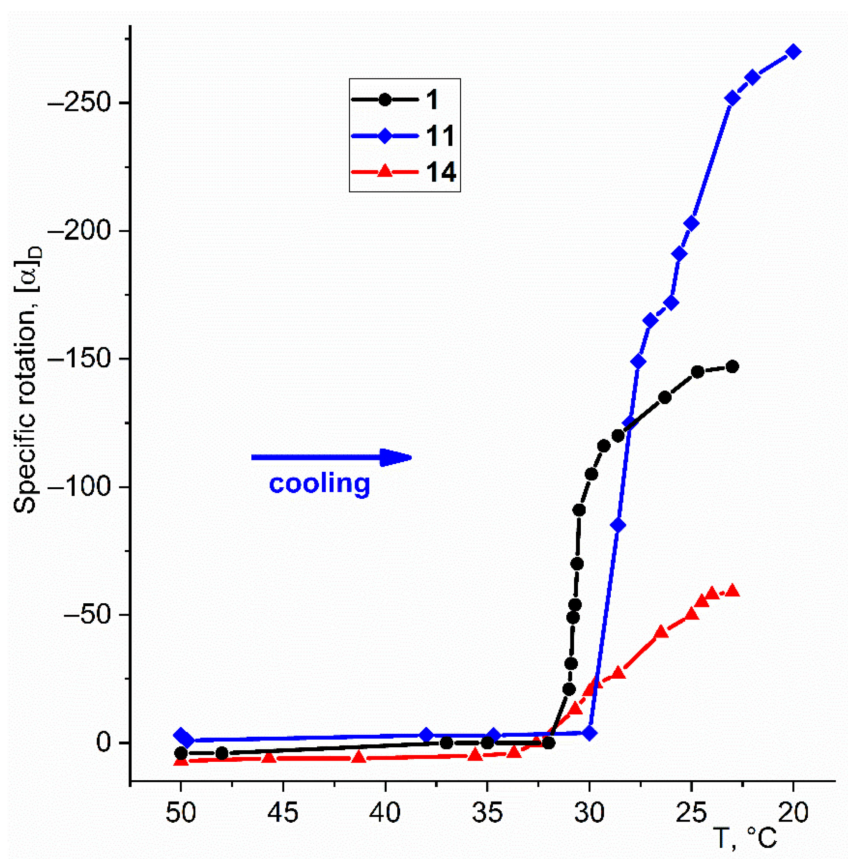

Figure 2. Changes in the specific rotation of transparent solutions of diol (S)-1, (S)-11 and $(S)-\mathbf{1 4}$ (c $0.078,0.22$ and $0.12 \mathrm{~g} / 100 \mathrm{~mL}$, correspondently) with decreasing temperature. Explanations in the text.

As can be seen from Figure 2, dramatic changes in the value of the specific rotation $[\alpha]_{D}$ from practically zero values for all three studied gelators occur in a rather narrow temperature interval of $32-27^{\circ} \mathrm{C}$. Apparently, this range could be considered as a characteristic one in which the network of fibrils, responsible for gel formation, forms. It should be noted that monotonic changes in $[\alpha]_{D}$ continue after reaching room temperature. For example, when the cell is kept at room temperature for a day, the specific rotation of the gel based on gelling agent 1 reaches the value $[\alpha]_{D}=-268$ (Table S1, line 2). Obviously, this is due to a kind of "ripening" of the gel, accompanied by the development of the fibril system and the formation of additional nodes. This phenomenon is associated with a noticeable $\left(\sim 10{ }^{\circ} \mathrm{C}\right)$ hysteresis between the temperature of formation and the temperature of destruction of the supramolecular gel.

Returning to the formulation of the problem, let us pay attention to the fact that in all cases investigated in this work, only enantiopure samples were found to be gelators. As expected, the ability to gel formation in enantiopure samples of opposite enantiomers turns out to be equally pronounced; therefore, the molecular configurations are not specially mentioned in Table 1 and further in the text. Entries 3, 10 and 11 require a particular comment, since these compounds exhibit the property of spontaneous resolution during crystallization, that is, they, crystallizing from a racemic sample, form conglomerates of enantiopure crystals [47]. Since in the case of para-propyl and ortho-methyl derivatives 3 and 10, enantiopure samples are not prone to gelation, such a tendency can hardly be expected from a mixture of enantiomers. However, in the case of ortho-ethyl derivative 11, enantiopure samples exhibit this property. In this case, the usual appeal to the lower solubility and greater stability of the crystalline racemate is inappropriate, since the energies of the crystalline packing of the enantiomers are the same, and the solubility of the racemic conglomerate, according to Meyerhoffer's rule [48], is approximately twice that for the pure enantiomer, which means that the gelling concentration is easily achieved for both $R$ and $S$ components.

In our opinion, in this and similar cases, gelation is hampered by the entropy factor, since the formation of enantiopure associates (fibrils) in a liquid racemic medium should compensate for the entropy of mixing of enantiomers (Rln2), which is absent in an enantiopure medium. In this respect, the gelation process is similar to the stereose- 
lective crystallization of enantiomers, which underlies the direct resolution of racemates by entrainment procedure $[47,49]$. As a rule, preparative stereoselective crystallization of pure racemates proceeds more efficiently if it is stimulated by enriching the initial sample with one of the enantiomers and/or by seeding the pure enantiomer. Similarly, an increase in the enantiomeric excess of 11 (starting from ee 16\%) also provokes the formation of a supramolecular gel.

A supramolecular gel has both the properties of a liquid (isotropy) and a solid (modulus of elasticity), and in this respect it is a mesophase. Liquid crystals are a classic example of a mesophase. There are numerous examples in the literature when a substance exhibits the property of a liquid crystal and, at the same time, can act as a gelator [50]. Like gels, liquid crystal mesophases exhibit unique chiral-dependent properties, which in itself is a progressive area of research [50,51]. However, it is unlikely that the driving forces responsible for the formation of both mesophases are of the same nature. Otherwise, it is not clear why, in the melts of compounds 3-9, shown in Scheme 1, liquid crystal phases are registered, which have similar properties for both enantiopure and racemic samples [38,39]. That said, supramolecular gelators in this set are only enantiopure samples of ethers 4-7.

The solution from which the gel is formed is an isotropic system. The gel that appears under certain circumstances turns out to be isotropic at the macrolevel, but significantly anisotropic at the microlevel of high aspect ratio fibrils penetrating it. The final stage in the evolution of the binary system "gelator/solvent" is a crystal, which is anisotropic by definition. In principle, the question of the relationship between the nature of the fibril anisotropy and the crystal anisotropy remains open. Some researchers believe that "for gels, studies show that gelation is effectively a crystallization event, with growth only occurring in one dimension" [52]. Other authors believe that "there is no reason to necessary assume that there is a link between crystallization and gelation" [8].

Bearing in mind the above-quoted statement by Richard Weiss about the individuality of each supramolecular gel, we can assume that in reality both these and other extremes are realized. In our further constructions, we will adhere to the following provisions. Firstly, it seems to us that the more pronounced the tendency towards crystallization of the gelator itself, the more reason to expect manifestations of this property in the gels formed by it. Secondly, Parthasarathi Dastidar believes that if the PXRD patterns of the xerogel and the bulk crystals of the gelator are near-superimposable, then this serves as reliable evidence of the common crystal organization of the self-assembled fibrils nets (SAFIN) and the gelator crystals [14]. Fully sharing this point of view, in the next section we will compare the corresponding powder patterns for all compounds that have shown a gelling ability.

\subsection{PXRD Results for Some Supramolecular Gels and Gelators}

Previously, xerogels formed during the degradation of gels based on the simplest LMWG scal-1 have already been studied by powder diffractometry (Figure 13 in ref. [35]). It was also noted there that the diffractograms of xerogels are similar to the experimental powder patterns for enantiopure crystalline samples, as well as with the curves calculated for the same samples from single crystal XRD data. In this paper, using the example of LMWGs 4-7, 11, 12, and 14, we investigated this process in more detail.

A typical set of diffraction patterns obtained by tracking the evolution of a freshly prepared gel based on the ortho-ethyl derivative $(R)-\mathbf{1 1}$ is shown in Figure 3. Already in the first diffractogram of the gel (curve 2), in addition to the natural amorphous halo, diffraction peaks are observed in the range of scattering angles $6.5^{\circ}, 12-13^{\circ}$ and $20-21^{\circ}$, indicating the presence of a crystalline phase in the sample. Thus, the initial stage of the transition from a gel-like state to a xerogel appears in the sample. As the solvent evaporates, the amorphous halo disappears and the intensity of the crystalline phase peaks increases. Comparison of the diffraction pattern with the experimental powder diffractogram of the enantiopure sample (curve 6) and the diffractogram simulated from single crystal XRD data of the enantiopure compound (curve 7) proves that the xerogel formed corresponds to the crystalline phase of the enantiopure sample. 


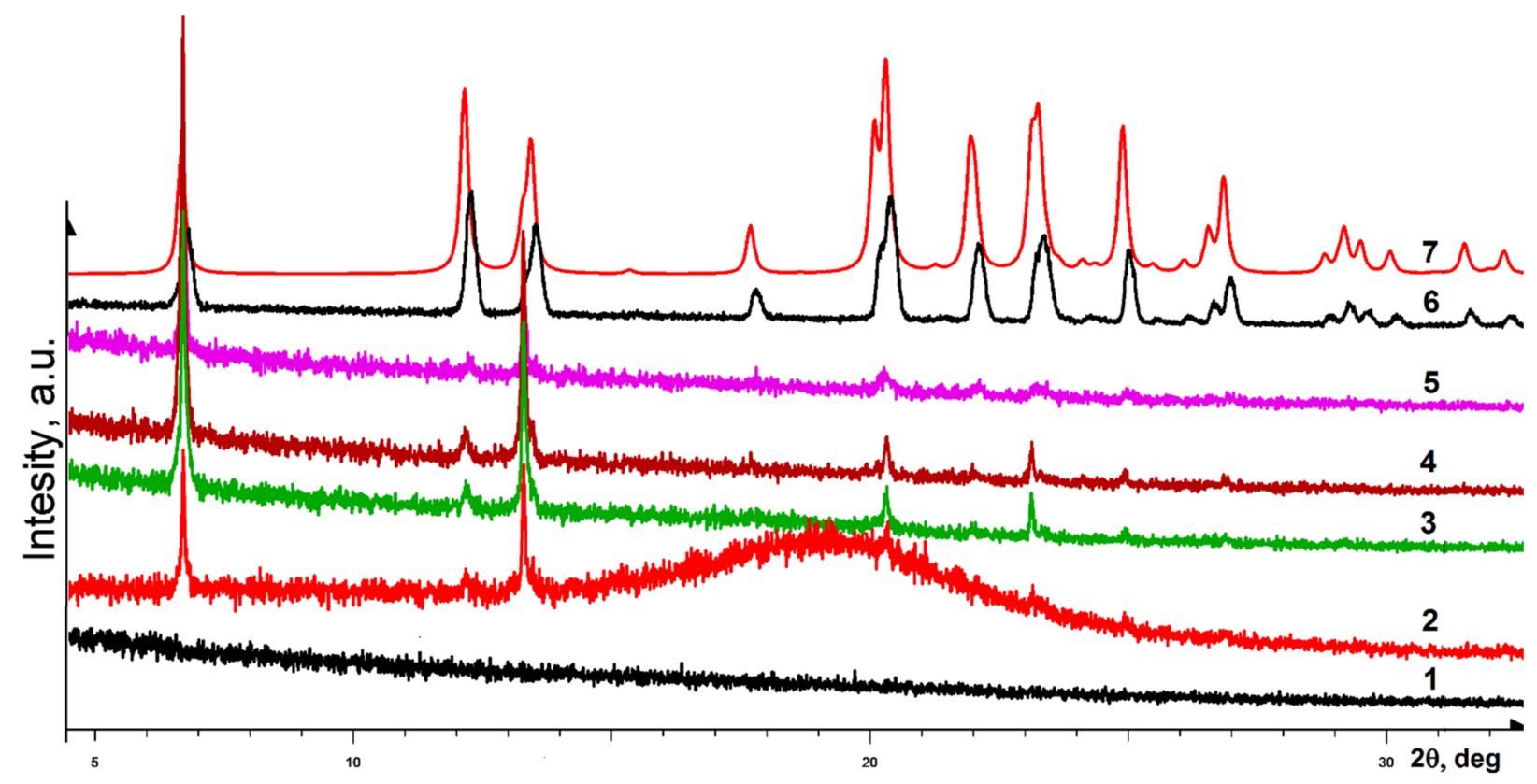

Figure 3. Powder diffraction patterns (bottom-up): (1) background scattering of a pure silicon plate; (2-4) evolution of the diffraction of (R)-11-based gel as the solvent naturally evaporates; (5) xerogel diffraction pattern after removing texturing from the sample; (6) experimental diffraction pattern of a polycrystalline enantiopure sample $(R)-11 ;(7)$ theoretical diffraction pattern calculated from single crystal XRD data for diol $(R)$-11. For clarity, the curves are vertically shifted relative to each other along the intensity axis.

The presence of strong interference peaks in the diffractogram of the initial gel, corresponding to reflections from the (002) and (004) planes, and their subsequent intensive growth indicate not only the orientational crystallization of the sample on the surface of the silicon plate, but also the features and morphology of the process of crystallization of supramolecular associates. Thus, the interplanar distance $\mathrm{d}_{002}$ corresponds to the thickness of the 2D bilayer supramolecular structure formed in the crystals of this sample due to the system of classical hydrogen bonds. The presence of higher orders of this reflection indicates a noticeable internal ordering of such bilayers even in the absence of ordering between such bilayers in the entire sample.

Figure 4 illustrates the transformation of gel based on 3,5-dimethyl derivative $(R)-\mathbf{1 4}$. The gels based on this compound are one of the most transparent and stable. In the early stages of drying (curves 1-3), there is no crystalline phase in the gel. Moreover, its appearance at the final stages of the destruction of the gel is marked by the small size of the crystallites and a low degree of crystallinity in general. Comparison of the diffraction peaks with the experimental ones in the powder diffractogram of the enantiopure sample (curve 8) and with the powder diffraction pattern of the racemate calculated on the basis of single crystal data (curve 9) indicates that the resulting crystalline phase corresponds to the enantiopure sample. A small amount of the crystalline phase formed from a noticeable gel volume (curve 7) is a consequence of the high holding capacity of this LMWG in relation to solvent molecules.

Figure 5 shows powder diffraction patterns related to a gel based on para-pentylsubstituted diol (R)-5. Already in the first diffractogram of the gel (Figure 5, curve 2), in addition to the amorphous halo, diffraction peaks are observed in the range of scattering angles $3,5,10,14$, and 18-20, indicating the presence of a crystalline phase in the sample. As the gel dries, the disappearance of the amorphous peak and an increase in the intensity of the peaks of the crystalline phase are observed. The crystallization on the surface of monocrystalline silicon occurs in an oriented manner, as evidenced by a sharp increase in the intensity of reflections from a series of basal planes. Disordering of the dried sample on the surface of the plate (curve 9) leads to a noticeable elimination of texturing 
and equalization of the intensities of reflections. Comparison of the observed diffraction peaks with the diffractogram simulated from single crystal data for sample $(R)-5$ (curve 1 ) indicates that the nature of the formed xerogel corresponds to the enantiopure crystalline phase. The racemic phase (compare curve 1) is practically absent in the final sample.

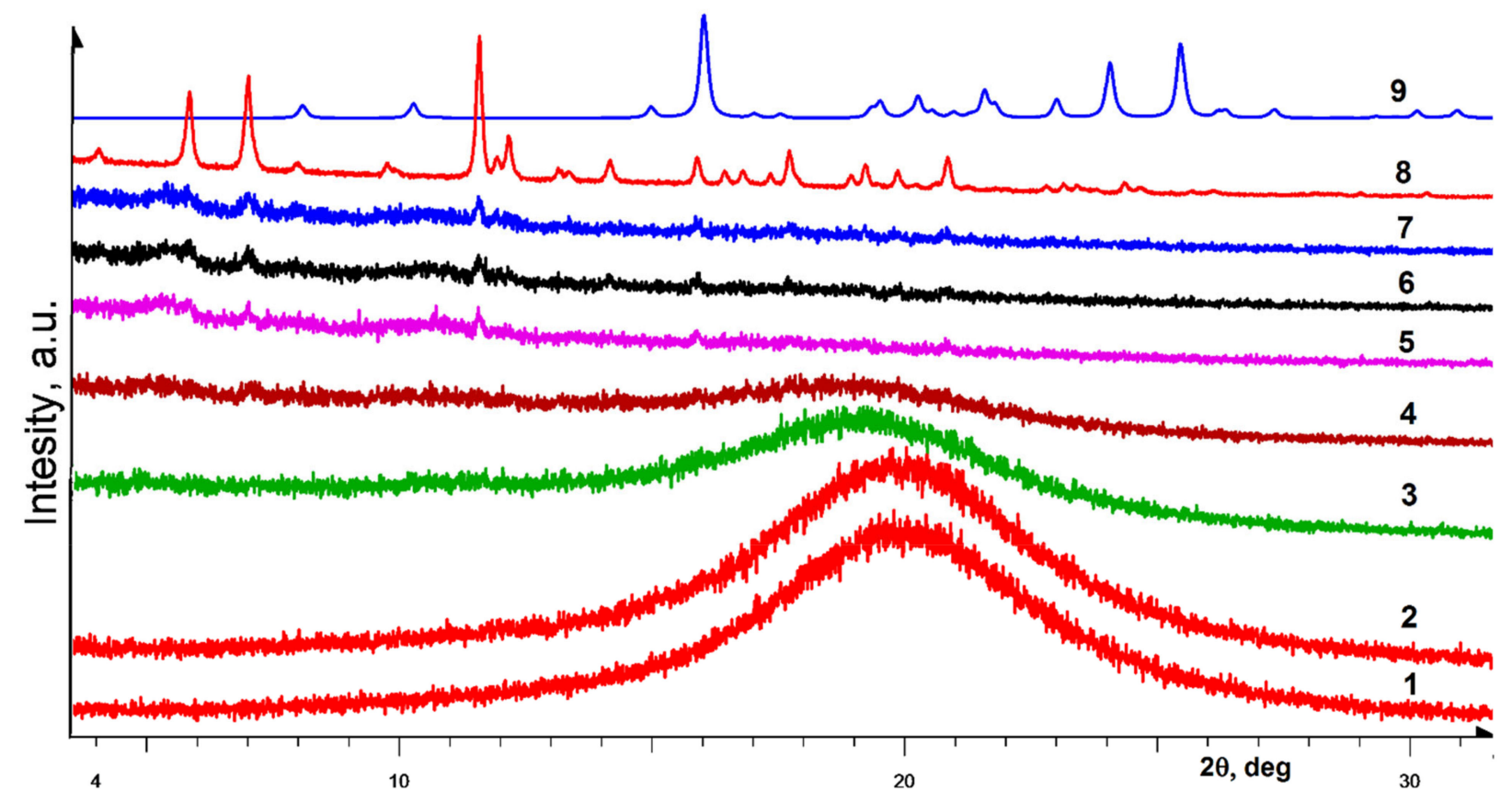

Figure 4. Powder diffraction patterns (bottom-up): (1-7) evolution of the diffraction of a gel based on (R)-14 as the solvent naturally evaporates; (8) experimental diffraction pattern of a polycrystalline enantiopure sample $(R)-14 ;(9)$ theoretical diffraction pattern calculated from single crystal XRD data for rac-14 diol.

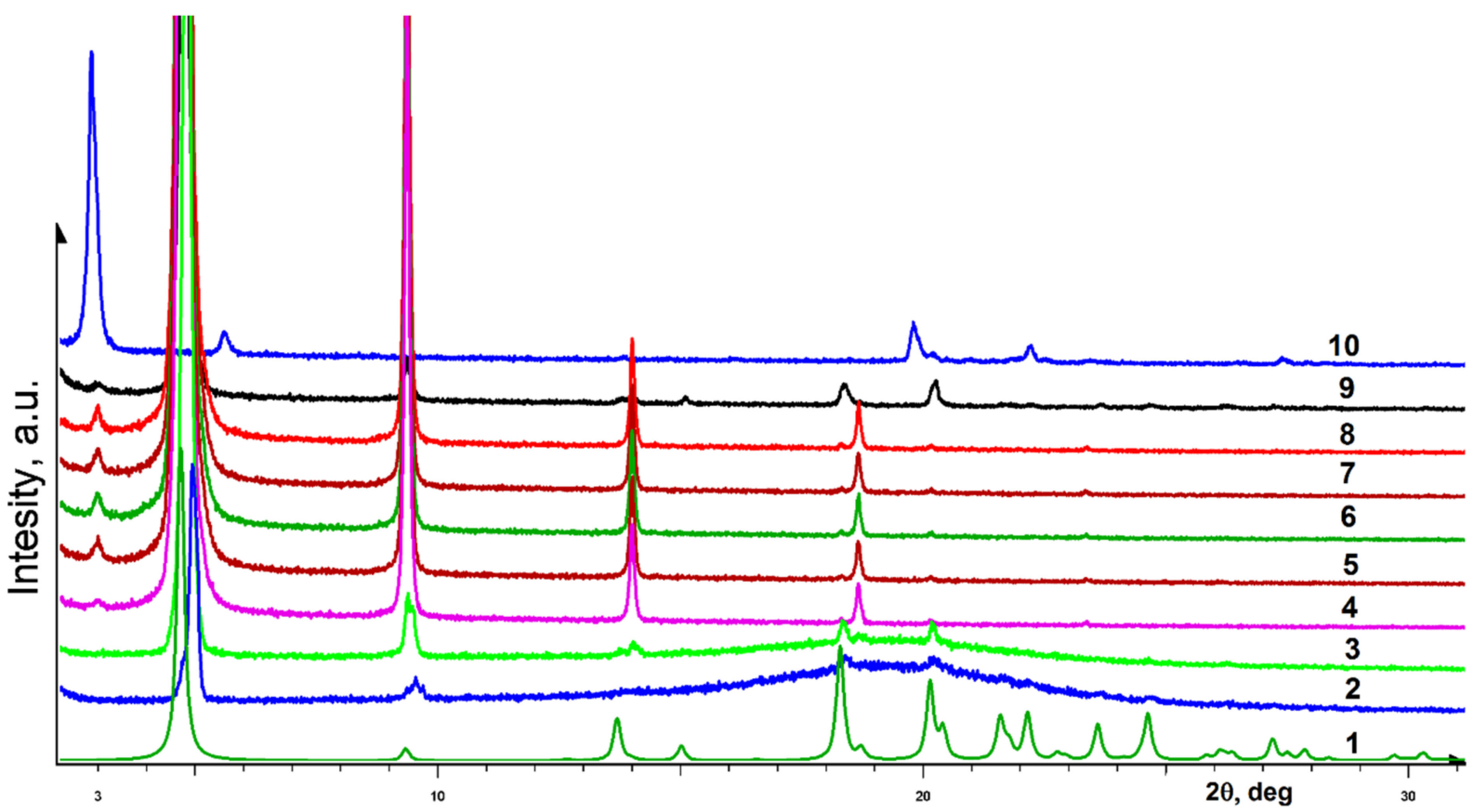

Figure 5. Powder diffraction patterns (bottom-up): (1) theoretical diffractogram calculated from single crystal XRD data for diol (R)-5; (2-8) evolution of (R)-5 based gel diffraction as the solvent naturally evaporates; (9) xerogel diffraction pattern after elimination of sample texturing; (10) experimental diffraction pattern of a rac-5 polycrystalline sample. 
The PXRD study of the evolution of gels, formed in nonane by $(R)-4,(R)-6,(R)-7$ and $(R)-\mathbf{1 2}$ gelators, is discussed in detail in the Supplementary Materials (Figures S1-S4 and comments to them). For all the cases we investigated, it was shown that the peaks present in the final powder diffraction patterns of xerogels were also present in the powder patterns of bulk crystals of enantiomeric prototypes, as well as in the powder patterns calculated from the results of SC-XRD for scalemic single crystals. Moreover, for all eight samples, all signals arising during the maturation of the xerogel were retained in the final PXR diffraction patterns, which indicates the absence of any transitional crystalline forms.

In the next section, we focus on the crystal packing features of compounds 1-14 that promote or prevent the formation of supramolecular gels.

\subsection{Single Crystals XRD Results and Crystal-Formative Motifs for Compounds Studied}

A valuable feature of the set we studied is that for all but one of its representatives we were able to establish the crystal structure of enantiopure samples. The specificity of crystals, which are intertwined threads and/or very thin needles (Figure 1b), did not allow us to solve this problem for ether $(R)-\mathbf{1 4}$. In contrast, the quality of rac-14 crystals (Figure 1c) was found to be satisfactory for SC-XRD studies. In the series of racemic crystals, we were unable to establish the crystal structure for ethers 5-9, the crystals of which are the finest mica-like plates. We also showed that compounds 3, 10, and 11 undergo spontaneous resolution, and their racemic samples contain only enantiopure crystals. Thus, if we do not take into account the problems of polymorphism and mirror symmetry, we have completely characterized eight out of 14 target compounds, and 22 out of 28 principally expected crystal structures are described.

The experimental details of X-ray structural analysis for previously investigated compounds are given in the works cited in Table 1, and for the compounds studied in this work are given in Tables S2-S4.

Hoping to ultimately identify the relationship between the crystal structure and the gelling ability of particular samples, we have turned to an analysis of the crystal-formative motifs present in them. For polyols, to which the aromatic glycerol ethers 1-14 belong, it is natural to expect that the main directional crystal-forming interactions will be the intermolecular hydrogen bonds (IMHB) O-H $\cdots \mathrm{O}-\mathrm{H}$. The corresponding fragment, titled "alcohol homosynthon", is included in the list of 25 commonly studied and utilized in crystal design synthons, cited in the well-known review by Corpinot and Bučar (Figure 8 in Ref. [53]), but at the same time, this synthon turns out to be the only one, more detailed information about which is not there presented.

A feature of our studied molecules is the presence of two hydroxyl groups that are different in position (terminal and middle) and in chemical nature (primary and secondary). In the absence of specific substituents that are able to independently participate in hydrogen bonding as a donor or acceptor, all variants of IMHB in our series are reduced to the interaction of the primary $\left(\mathrm{O}_{1}-\mathrm{H}_{1}\right)$ or secondary $\left(\mathrm{O}_{2}-\mathrm{H}_{2}\right)$ donor with one of the three acceptors, $\mathrm{O}_{1}, \mathrm{O}_{2}$ or $\mathrm{O}_{3}$ atoms (see Scheme 2 for the numbering).

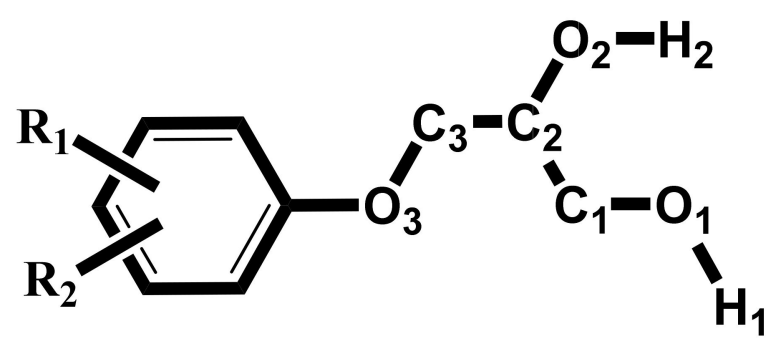

Scheme 2. Partial atom numbering in glycerol fragment of molecules 1-14.

We begin our analysis with the scalemic samples of diols 5-9 and racemic diol 14, first studied by SC-XRD in this work. Figure 6 shows a fragment of a supramolecular motif realized in (R)-5 crystals. The primary element of such a motif, which gives it integrity, is 
a series of endless helical sequences of intermolecular hydrogen bonds that form around the screw axes $2_{1}$ parallel to the $0 b$ direction. In Figure $6 a$, these spirals are marked with blue circles. Their helical nature is clearly visible from Figure $6 \mathrm{~b}$. The construction of a complete helix turn requires the participation of four molecules, each of which provides for this purpose one of the two O-H fragments available in the molecule. Unclaimed hydroxyl groups are involved in the construction of adjacent helixes. Taken together, the combination of rigid elements, united by a system of flexible spacers, forms a 2D bilayer parallel to the $0 a b$ plane (Figure 6a). This motif, hereinafter referred to as BL-1, with certain modifications, is often found in crystals of the studied set of compounds (Table 1). In particular, it is realized for the ether $(R)-7$, newly investigated in this work (Figure S5).
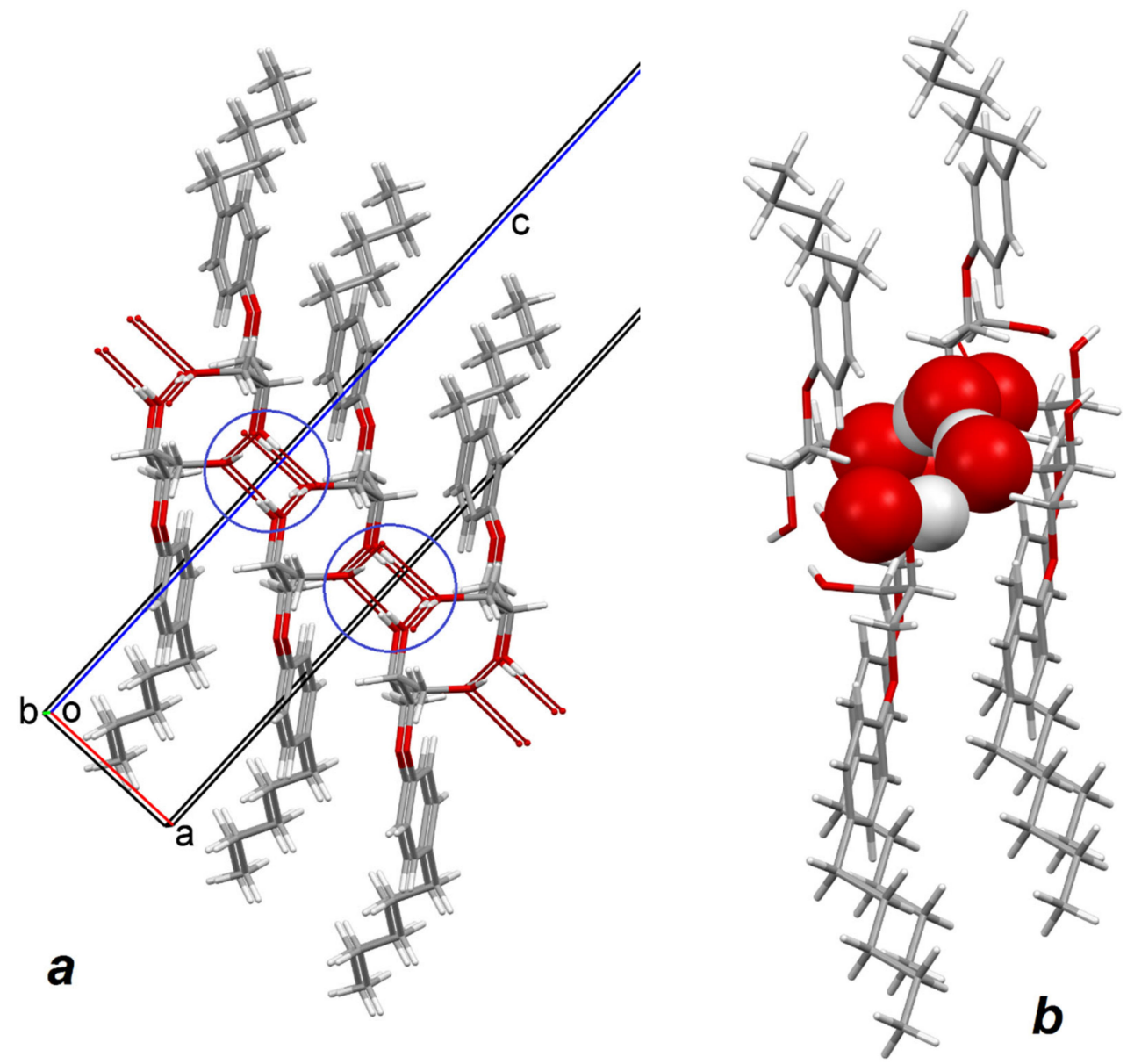

Figure 6. System of intermolecular hydrogen bonds in (R)-5 crystals. Formation of 2D bilayer parallel 0ab plane (a) and $M$-helix formed by intermolecular H-bonds around screw axis $2_{1}$ parallel $0 b$ direction (b).

Diol $(R)-6$ crystals are formed with the participation of two independent molecules in an asymmetric unit of the unit cell. In Figure 7, the independent A molecules are marked in green and the B molecules in blue. As can be seen from Figure 7a, in this case the main fastening elements of the supramolecular crystal-formative motif are endless spiral sequences (chains) of intermolecular H-bonds formed around axes $2_{1}$. Molecules A and B take part in them on unequal rights. Molecules A participate in their construction by both hydroxyl groups, while $\mathrm{B}$ molecules transfer only secondary $\mathrm{OH}$ groups for this purpose. Primary B molecules hydroxyls participate in the construction of an almost flat zigzag chain oriented around another screw axis 21 parallel $0 b$ direction (Figure $7 b$ ). Note that the 2D bilayer formed in this way (we denote it by the abbreviation BL-2) turns out to be even more flexible than BL-1, since the stiffeners in it are separated by a longer and, therefore, more flexible spacer. We identified exactly the same motif in $(R)-8$ crystals (Figures S6 and S7). 

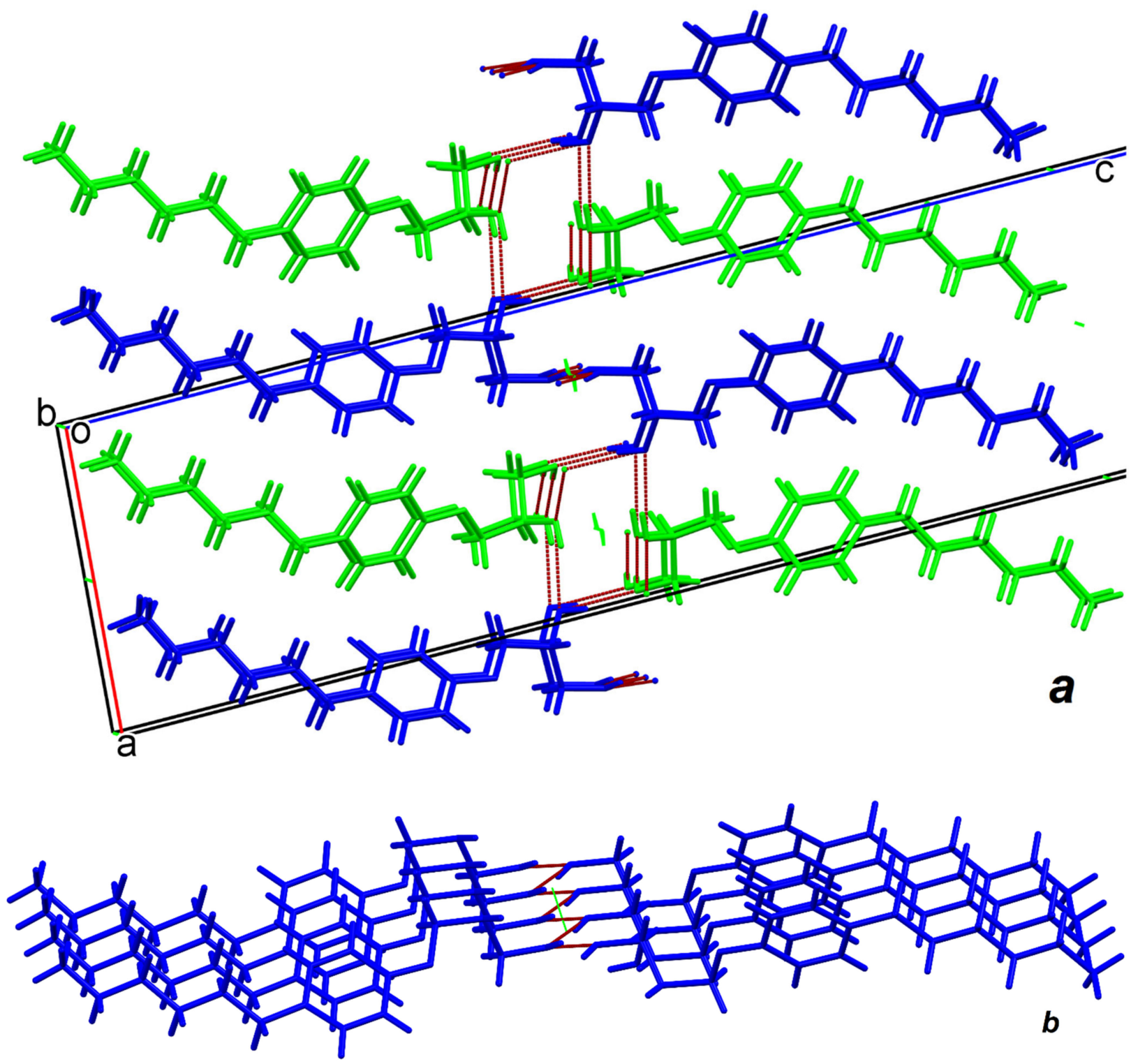

Figure 7. System of intermolecular hydrogen bonds in (R)-6 crystals. Symmetry independent A molecules are marked in green and B molecules in blue. Formation of 2D bilayer parallel 0ab plane (a) and almost flat zigzag chain formed by intermolecular $\mathrm{H}$-bonds around screw axis 21 parallel $0 b$ direction (b).

Comparison of the characteristics of the unit cells for the ethers (R)-8 and (R)-9 (Table S2) shows that, while the space group $P 2_{1}$ and two symmetry independent molecules are maintaining for both compounds, owing to elongation of alkyl substituent of one methylene unit, the character of the packing is changed again. Figure 8 shows a fragment of the crystal packing, which corresponds to the primary supramolecular motif in the (R)-9 crystals. Shown in the Figure 1D column is developed around the screw axis $2_{1}$. These columns are formed by two pairwise symmetric systems of intermolecular H-bonds.

The IMHB system implemented in crystals $(R)-\mathbf{9}$ is described in more detail in the Supplementary Materials (Figures S8 and S9). Here we note that this motif, a one-dimensional column, which we will designate with the abbreviation CL-1, is a rigid structure, fastened by at least three spiral chains that have common elements.

The last substance whose crystal structure was studied in this work is the racemate of diol 14. Figure 9 illustrates the main details of the organization of the supramolecular crystal-formative motif for this sample. 


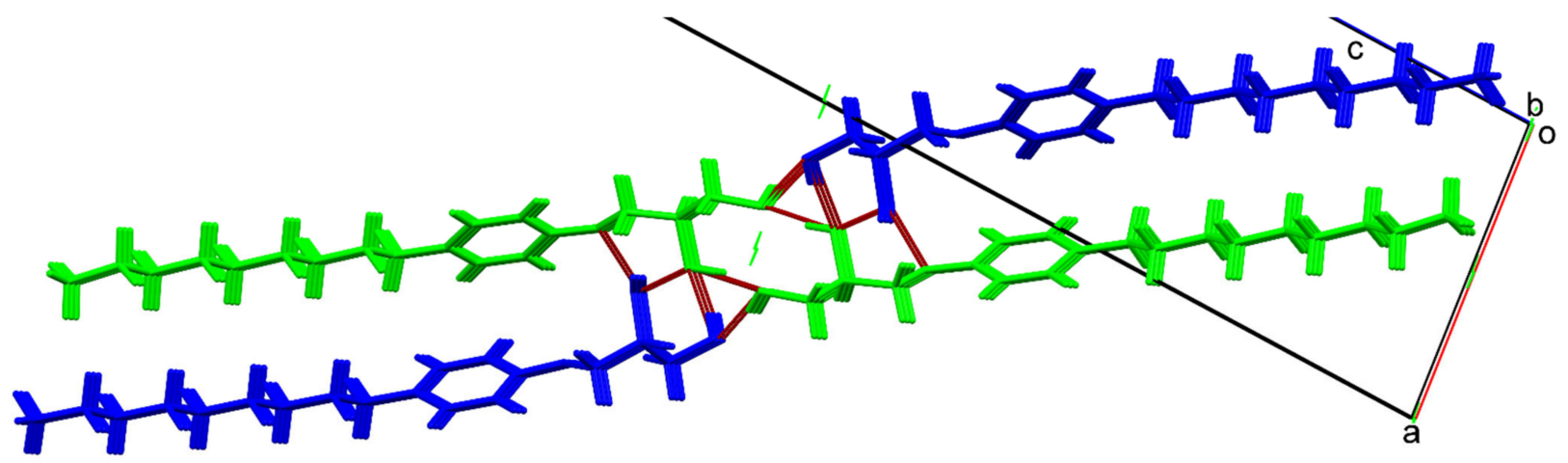

Figure 8. Details of $(R)-9$ crystal packing; independent A molecules are colored in green, independent B molecules are colored in blue. Red dotted lines mark intermolecular hydrogen bonds. View along the $0 b$ axis.

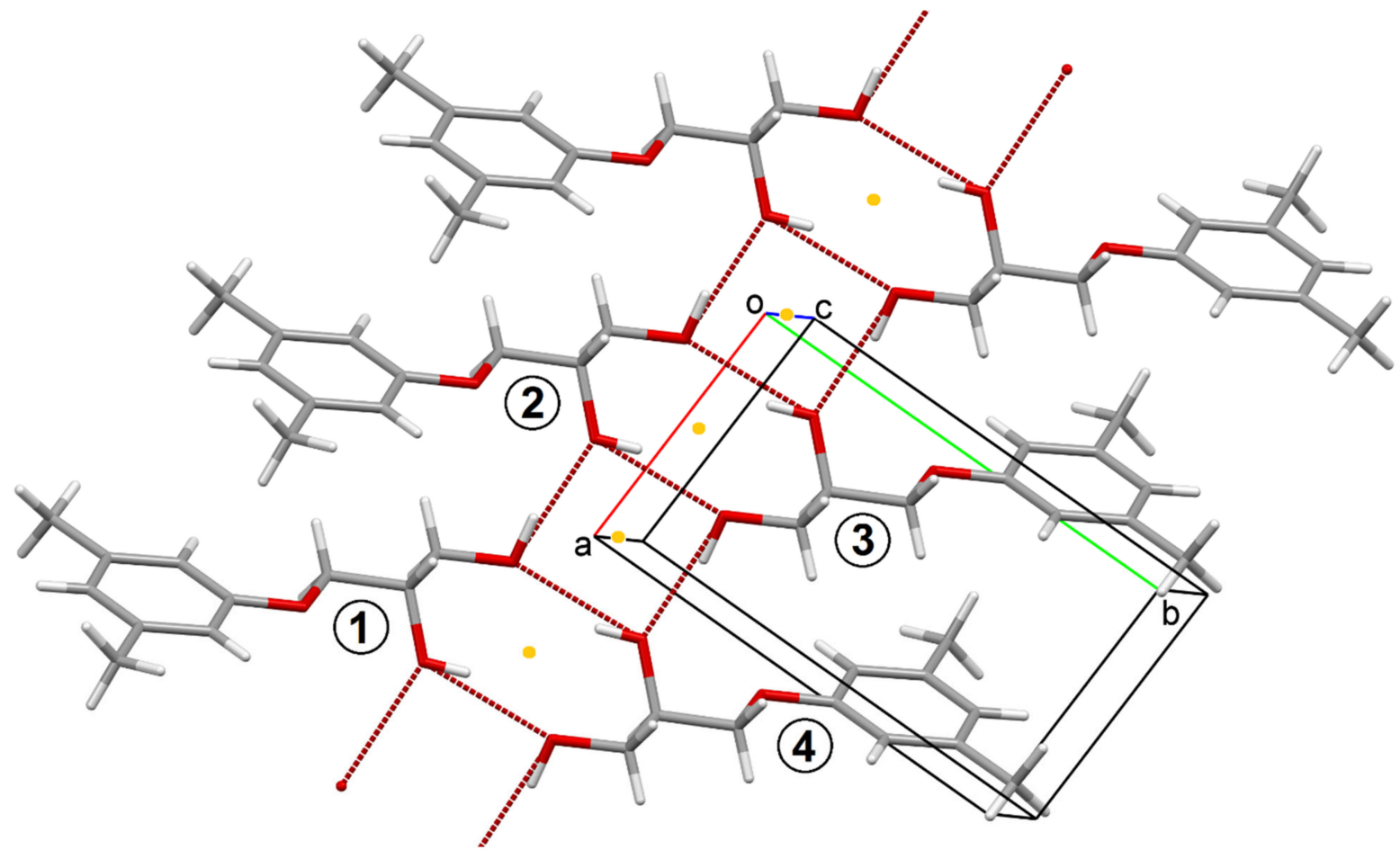

Figure 9. Details of rac-14 crystal packing. Red dotted lines mark intermolecular hydrogen bonds; orange circles indicate inversion centers. The numbers 1-4 in circles represent the molecules discussed in the text.

It can be seen from the figure that the main element of the motif is the centrosymmetric ring $\mathbf{R}_{\mathbf{4}}{ }^{4}(\mathbf{8})$, for which molecules 1 and 3 provide their primary hydroxyl groups $\mathrm{O}_{1}-\mathrm{H}_{1}$, and molecules 2 and 4 provide secondary hydroxyls $\mathrm{O}_{2}-\mathrm{H}_{2}$. The remaining free hydroxyl groups form the same rings grouped along the $0 a$ direction. In general, the motif is a one-dimensional ribbon, which we will designate with the RR symbol (ribbon of rings).

Another one-dimensional motif, which is a column held together by a unified helical sequence of IMHB, characterizes the packing of a non-racemic sample of the meta-methyl derivative $(R)-\mathbf{1 3}$, and is also realized in any samples of the drug mefenesin 10 [46]. In Table 1 , this motif is designated by the symbol CL-2.

Identical primary supramolecular motifs in rac-1 and rac-13 crystals were discussed in detail by us earlier [35]. It is clear from the figures and the discussion in the text that these motifs are rigid $2 \mathrm{D}$ bilayers connected by a dense network of intermolecular $\mathrm{H}$-bonds, formed by glide planes present in the lattice. In Table 1, such bilayers are designated as BL-3. The primary supramolecular crystal-formative motif in rac-2 crystals is even more 
rigid. Like the two previous ones, it is formed by a glide plane. A sophisticated way of constructing it with the participation of all four independent molecules present in the asymmetric unit is illustrated in Figure 5 in Ref. [39]. In Table 1, this motif is designated as BL-4.

\subsection{Crystal Organization vs. Gelation Abilities}

As follows from Table 1, in the samples of compounds 1-14 studied by the SC-XRD method, seven types of supramolecular crystal-formative motifs are realized, of which three are one-dimensional and four are two-dimensional. At the same time, only the BL-1 (Figure 6) and BL-2 (Figure 7) motifs are found in the samples exhibiting LMWG properties, and at this stage of the study, it is necessary to answer the question: what qualities of these two motifs distinguish them from the other five unclaimed in the processes of gelation?

The first, objective, we believe, is the dimensionality of the motive. For obvious reasons, substances that form closed zero-dimensional supramolecular motifs should be excluded from the list of potential low molecular weight organogelators. Compounds, forming 3D hydrogen bond nets as a primary crystal-formative motif, in our opinion, are also not capable of gelation. There is no reason to believe that a substance prone to bulk binding in crystals will be prone to the formation of linear structures (fibrils) in solution. Further, we are not aware of examples of LMWGs that form isolated one-dimensional crystal-forming motifs in crystals. Apparently, a one-dimensional chain of molecules, held together only by ordinary intermolecular hydrogen bonds, whose energy lies in the range of 8-22 $\mathrm{kJ} \mathrm{mole}^{-1}$ [54] and is comparable in order of magnitude with the energy of Brownian motion, cannot form a network capable of immobilizing orders of magnitude exceeding the amount of solvent. In most publications on the topic under discussion, a bilayer is explicitly or implicitly accepted as the initial nanoassociate for building a gel.

The available literature provides numerous theoretically and experimentally confirmed examples of the transformation of flat primary nanoassociates, involved in gelation, into twisted ribbons, single-walled and multilayer nanotubes, which serve as SAFIN elements immobilizing liquid $[11,12]$. Consequently, the second essential quality of a sufficiently durable initial associate (in relation to the discussed set of gelators, we consider that this is the primary crystal-formative bilayer motif) is its flexibility. We noted above that of the four types of bilayers identified in crystals 1-14, only BL-1 and BL-2 have this property.

Apparently, the combination of strength and flexibility of the primary motif is a significant, possibly necessary, but not sufficient prerequisite for gelation in solutions, and, before moving on, it is worth indicating the reasons why compounds crystallizing by this type do not form gels. In our case, this applies to samples scal-2, rac-3, scal-3, rac-4, scal-8 and rac-11. The formation of supramolecular gels depends on many factors, of which solubility is perhaps the most studied [13]. It is known that gelation in a given solvent (individual or mixed) occurs in a fairly narrow range of solubility. At the same time, going beyond its limits, both in one and the other direction, prevents gelation. Our quantitative data on the solubility of some alkylphenyl ethers of glycerol in cyclohexane are given in the Supplementary Materials (Table S5). It follows from these data that gelation, when it occurs, occurs in a narrow concentration range $1.2<\mathrm{C}_{\text {gel }}\left(\mathrm{mmol} \mathrm{L}^{-1}\right)<1.9$. In our case, the scal-2 sample is too much soluble, and the rac-3, scal-3, and scal-8 samples are too poorly soluble to form gels. The peculiarities of gelling of racemic samples of diol 11, which is a conglomerate, are due to kinetic factors, as discussed in detail in Section 3.1.

There is no doubt that solubility plays an important role in the processes of supramolecular gelation, and by varying the solvent, it is possible to help a substance, capable of gelation, to realize these potencies [13], but we believe that the very ability to form a gel is associated primarily with the nature of the substance. For alkyl-substituted phenyl ethers of glycerol, we have shown that supramolecular gelation is associated, firstly, with the bilayer character and, secondly, with the flexibility of the primary crystal-forming motif of the initial crystalline sample. 
Infinite bilayer structures in crystals cannot be formed by finite elements of symmetry, such as inversion centers, planes, and simple rotational axes. In the overwhelming majority of cases, they are formed with the participation of glide planes or screw axes, and at this point, the fundamental difference between racemic and enantiopure samples of a chiral substance becomes obvious: glide planes are absent in crystals of enantiopure samples, and molecules with opposite configurations present in the racemate cannot (strictly speaking, experience significant difficulties) participate in the formation of continuously extended chains of intermolecular hydrogen bonds around the screw axes.

We believe that the dominant role of the screw axes in the formation of crystals of enantiopure compounds is the final reason responsible for the predominance of enantiopure gelator compounds over racemic ones. At least this is true for low molecular weight compounds, the crystal-formative motifs of which are due to intermolecular hydrogen bonds.

Note here that for such compounds, the formation of infinite bilayers around the screw axes is possible only under the condition that several belonging to each molecule's functional groups, able to form intermolecular hydrogen bonds, are not involved in a one-dimensional helical sequence in a complete set (in this case, a one-dimensional motif will form). On the contrary, they must be spaced apart along adjacent spiral rods. In its turn, this means that the dominance of the screw axes also provides the second necessary condition for gelation - the flexibility of the bilayer, since, in order to maintain the integrity of the motif, the rigid spiral rods of the IMHB will be connected by flexible spacers from a more or less extended sequence of covalent bonds.

The direct influence of the chirality of a sample on the predominance of one or another supramolecular crystal-formative motif in its crystals was previously noted by us using the example of the amide synthon $\mathrm{O}=\mathrm{C}-\mathrm{N}-\mathrm{H}$ [55]. We believe that the results of this study add another, somewhat exotic, evidence of the importance of accounting for this factor.

\section{Conclusions}

Out of 14 pairs of racemic and enantiopure samples of alkyl-substituted phenyl ethers of glycerol (Scheme 1) the stable gels in a hydrocarbon medium (in nonane) form only eight enantiopure diols 1, 4-7, 11, 12, 14. Stability and high transparency of gels based on para-methyl-, ortho-ethyl-, and 3,5-dimethylphenyl ethers of glycerol 1, 11 and 14 made it possible for the first time to investigate the formation of gels from solutions by polarimetry method. Of 28 potentially possible crystalline samples, 22 were studied by single crystal X-ray diffraction, of which crystalline samples of enantiopure para-n-alkylphenyl glycerol ethers [alkyl = pentyl (5), hexyl (6), heptyl (7), octyl (8), nonyl (9)] and racemic 3-(3,5dimethylphenoxy)propane-1,2-diol (rac-14) have been examined in this work.

The set of freshly prepared gels as well as the process of their transformation into xerogels as the solvent evaporates was studied by powder X-ray diffractometry. It was shown that the peaks present in the final powder diffraction patterns of xerogels were also present in the powder patterns of bulk crystals of enantiomeric prototypes, as well as in the powder patterns calculated from the results of SC-XRD for scalemic crystals. Hence, one can conclude that the structure of the fibrils that form the gel is based on the crystalline structure (at least, a supramolecular crystal-formative motif) of a pure gelator.

In 22 samples of compounds 1-14 studied by single crystal X-ray diffraction, seven different types of supramolecular crystal-forming motifs were realized, including four bilayer ones, of which only two were realized in the crystals of supramolecular gelators. The bilayer motifs BL-3 and BL-4, realized in rac-1, rac-13 and rac-2 crystals, were described in detail by us earlier $[35,39]$. Being organized by glide planes, which is typical for racemic crystals, such bilayers are linked by a two-dimensional network of intermolecular hydrogen bonds $\cdots \mathrm{O}-\mathrm{H} \cdots \mathrm{O}-\mathrm{H} \cdots$, which gives them a rigid character.

In crystals of enantiopure supramolecular gelators, there were two other types of bilayers, designated by us as BL-1 (Figure 6) and BL-2 (Figure 7). In both cases, screw axes $2_{1}$ acted as infinite symmetry elements that create a bilayer structure. Rigidity was given to the motifs by relatively isolated infinite spiral sequences of the same hydrogen bonds 
organized around such axes. In both cases, these stiffeners were linked by sequences of covalent bonds that act as flexible spacers.

Apparently, the combination of the rigidity of the primary motif, which provides the physical strength of the primary supramolecular associates, sufficient for their preservation during the subsequent strengthening, as well as flexibility, which allows the bilayer associate to roll up, for example, into a multilayer nanotube, is a necessary condition for the formation of fibrils that retain the solvent in the gel. This combination is difficult to implement for a system of glide planes, typical infinite symmetry elements in racemate crystals, and relatively easy for a system of screw axes-the only infinite symmetry elements in enantiopure crystals. We believe that the dominant role of the screw axes in the formation of crystals of enantiopure compounds is the final reason responsible for the predominance of enantiopure gelators over racemic ones. At least this is true for low molecular weight compounds, the crystal-formative motifs of which are due to intermolecular hydrogen bonds. Further expansion of the sets of simple chiral compounds, for which, in parallel with the study of the peculiarities of the gelling ability, the details of the organization of their racemic and enantiopure crystals will be reliably established, will make it possible to test the reliability of the above hypothesis.

Supplementary Materials: The following are available online at https://www.mdpi.com/article/10 .3390/sym13040732/s1, Figures S1-S4: Powder XRD results for compound (R)-4, 6, 7, 12. Figures S5-S9: Details of (R)-7-9 crystal packing. Table S1: Specific rotation of gels formed by compounds 1, $\mathbf{1 1}$ and $\mathbf{1 4}$ in nonane. Tables S2-S4: Crystallographic summary of compounds (R)-5 -(R)-9 and rac-14. Table S5: Solubility data of some alkyl substituted phenyl glycerol ethers in cyclohexane.

Author Contributions: Conceptualization, writing-review and editing, A.A.B.; PXRD investigations, SC-XRD analysis of $r a c-14$, A.T.G.; methodology, samples preparation, proofreading the manuscript, Z.A.B.; SC-XRD experiments of compounds (R)-5-9, R.R.F. and O.A.L. All authors have read and agreed to the published version of the manuscript.

Funding: This research received no external funding.

Acknowledgments: X-ray diffraction data were registered on the equipment of the Assigned SpectralAnalytical Center of FRC Kazan Scientific Center of RAS. The authors thank D.V. Zakharychev for valuable technical assistance.

Conflicts of Interest: The authors declare no conflict of interest.

\section{References}

1. Azzaroni, O.; Ariga, K. Nanoarchitectonics, now. Mol. Syst. Des. Eng. 2019, 4, 9-10. [CrossRef]

2. Weiss, R.G. The past, present, and future of molecular gels. What is the status of the field, and where is it going? J. Am. Chem. Soc. 2014, 136, 7519-7530. [CrossRef]

3. Bacani, R.; Trindade, F.; Politi, M.J.; Triboni, E.R. (Eds.) Nano Design for Smart Gels; Elsevier: Amsterdam, The Netherlands, 2019; $262 p$.

4. Weiss, R.G. (Ed.) Molecular Gels: Structure and Dynamics; The Royal Society of Chemistry: Cambridge, UK, 2018; 392p.

5. Guenet, J.M. Organogels. Thermodynamics, Structure, Solvent Role, and Properties; Springer: Cham, Switzerland, 2016; 122p.

6. Escuder, B.; Miravet, J.F. (Eds.) Functional Molecular Gelators; The Royal Society of Chemistry: Cambridge, UK, 2014; 319p.

7. Zhang, J.; Hu, Y.; Li, Y. Supramolecular gels. In Gel Chemistry: Interactions, Structures and Properties; Lecture Notes Chemistry; Springer: Singapore, 2018; Volume 96, pp. 9-59. [CrossRef]

8. Draper, E.R.; Adams, D.J. Low-molecular-weight gels: The state of the art. Chem 2017, 3, 390-410. [CrossRef]

9. Draper, E.R.; Adams, D.J. How should multicomponent supramolecular gels be characterised? Chem. Soc. Rev. 2018, 47, 3395-3405. [CrossRef] [PubMed]

10. Yu, G.; Yan, X.; Han, C.; Huang, F. Characterization of supramolecular gels. Chem. Soc. Rev. 2013, 42, 6697-6722. [CrossRef] [PubMed]

11. Sang, Y.; Liu, M. Nanoarchitectonics through supramolecular gelation: Formation and switching of diverse nanostructures. Mol. Syst. Des. Eng. 2019, 4, 11-28. [CrossRef]

12. Chivers, P.R.A.; Smith, D.K. Shaping and structuring supramolecular gels. Nat. Rev. Mater. 2019, 4, 463-478. [CrossRef]

13. Lan, Y.; Corradini, M.G.; Weiss, R.G.; Raghavan, S.R.; Rogers, M.A. To gel or not to gel: Correlating molecular gelation with solvent parameters. Chem. Soc. Rev. 2015, 44, 6035-6058. [CrossRef] [PubMed]

14. Dastidar, P. Designing supramolecular gelators: Challenges, frustrations, and hopes. Gels 2019, 5, 15. [CrossRef] 
15. Mehwish, N.; Dou, X.Q.; Feng, C.L. Trends in design of C-2-symmetric supramolecular chiral gelators. Eur. Polym. J. 2019, 117, 236-253. [CrossRef]

16. Liu, M.; Ouyang, G.; Niu, D.; Sang, Y. Supramolecular gelatons: Towards the design of molecular gels. Org. Chem. Front. 2018, 5, 2885-2900. [CrossRef]

17. Hoque, J.; Sangaj, N.; Varghese, S. Stimuli-responsive supramolecular hydrogels and their applications in regenerative medicine. Macromol. Biosci. 2019, 19, e1800259. [CrossRef]

18. Okesola, B.O.; Smith, D.K. Applying low-molecular weight supramolecular gelators in an environmental setting-Self-assembled gels as smart materials for pollutant removal. Chem. Soc. Rev. 2016, 45, 4226-4251. [CrossRef]

19. Skilling, K.J.; Citossi, F.; Bradshaw, T.D.; Ashford, M.; Kellam, B.; Marlow, M. Insights into low molecular mass organic gelators: A focus on drug delivery and tissue engineering applications. Soft Matter 2014, 10, 237-256. [CrossRef]

20. Weiss, R.G. Controlling variables in molecular gel science: How can we improve the state of the art? Gels 2018, 4, 25. [CrossRef] [PubMed]

21. Weiss, R.G. Introduction: An overview of the "What" and "Why" of molecular gels. In Molecular Gels: Structure and Dynamics, The Royal Society of Chemistry: Cambridge, UK, 2018; Chapter 1.

22. Frkanec, L.; Zinic, M. Chiral bis(amino acid)- and bis(amino alcohol)-oxalamide gelators. Gelation properties, self-assembly motifs and chirality effects. Chem. Commun. 2010, 46, 522-537. [CrossRef] [PubMed]

23. Brizard, A.; Oda, R.; Huc, I. Chirality effects in self-assembled fibrillar networks. Top. Curr. Chem. 2005, 256, 167-218. [CrossRef] [PubMed]

24. Qin, M.; Zhang, Y.; Xing, C.; Yang, L.; Zhao, C.; Dou, X.; Feng, C. Effect of stereochemistry on chirality and gelation properties of supramolecular self-assemblies. Chem. Eur. J. 2021, 27, 3119-3129. [CrossRef] [PubMed]

25. Kodama, K.; Kawamata, R.; Hirose, T. Synthesis and evaluation of chiral beta-amino acid-based low-molecular-weight organogelators possessing a methyl/trifluoromethyl side chain. New J. Chem. 2019, 43, 2882-2887. [CrossRef]

26. McAulay, K.; Dietrich, B.; Su, H.; Scott, M.T.; Rogers, S.; Al-Hilaly, Y.K.; Cui, H.; Serpell, L.C.; Seddon, A.M.; Draper, E.R.; et al. Using chirality to influence supramolecular gelation. Chem. Sci. 2019, 10, 7801-7806. [CrossRef] [PubMed]

27. Tomasson, D.A.; Ghosh, D.; Krzisnik, Z.; Fasolin, L.H.; Vicente, A.A.; Martin, A.D.; Thordarson, P.; Damodaran, K.K. Enhanced mechanical and thermal strength in mixed-enantiomers-based supramolecular gel. Langmuir 2018, 34, 12957-12967. [CrossRef]

28. Liu, M.; Zhang, L.; Wang, T. Supramolecular chirality in self-assembled systems. Chem. Rev. 2015, 115, 7304-7397. [CrossRef]

29. Duan, P.; Cao, H.; Zhang, L.; Liu, M. Gelation induced supramolecular chirality: Chirality transfer, amplification and application. Soft Matter 2014, 10, 5428-5448. [CrossRef] [PubMed]

30. Zhang, L.; Jin, Q.X.; Liu, M.H. Enantioselective recognition by chiral supramolecular gels. Chem. Asian J. 2016, 11, 2642-2649. [CrossRef]

31. Fuhrhop, J.H.; Schnieder, P.; Rosenberg, J.; Boekema, E. The chiral bilayer effect stabilizes micellar fibers. J. Am. Chem. Soc. 1987, 109, 3387-3390. [CrossRef]

32. Fuhrhop, A.H.; Wang, T.Y. Bolaamphiphiles. Chem. Rev. 2004, 104, 2901-2937. [CrossRef] [PubMed]

33. Kulkarni, C.; Berrocal, J.A.; Lutz, M.; Palmans, A.R.A.; Meijer, E.W. Directing the solid-state organization of racemates via structural mutation and solution-state assembly processes. J. Am. Chem. Soc. 2019, 141, 6302-6309. [CrossRef] [PubMed]

34. Bredikhin, A.A.; Bredikhina, Z.A.; Akhatova, F.S.; Gubaidullin, A.T. $p$-Tolyl glycerol ether: Is it possible to find more simple molecular organogelator with pronounced chirality driven properties? Chem. Commun. 2010, 46, 3523-3525. [CrossRef]

35. Bredikhin, A.A.; Zakharychev, D.V.; Bredikhina, Z.A.; Gubaidullin, A.T.; Fayzullin, R.R. Crystal structure and phase behavior of the tolyl glycerol ethers. From the conglomerate former to the chirality driven nanogelator. CrystEngComm 2012, 14, 211-222. [CrossRef]

36. Bredikhin, A.A.; Zakharychev, D.V.; Gubaidullin, A.T.; Bredikhina, Z.A. Solid phase behavior, polymorphism, and crystal structure features of chiral drug Metaxalone. Cryst. Grow. Des. 2018, 18, 6627-6639. [CrossRef]

37. Bredikhin, A.A.; Bredikhina, Z.A.; Novikova, V.G.; Pashagin, A.V.; Zakharychev, D.V.; Gubaidullin, A.T. Three different types of chirality driven crystallization within the series of uniformly substituted phenyl glycerol ethers. Chirality 2008, 20, $1092-1103$. [CrossRef]

38. Bredikhin, A.A.; Zakharychev, D.V.; Fayzullin, R.R.; Antonovich, O.A.; Pashagin, A.V.; Bredikhina, Z.A. Chiral para-alkyl phenyl ethers of glycerol: Synthesis and testing of chirality driven crystallization, liquid crystal, and gelating properties. Tetrahedron Asymmetry 2013, 24, 807-816. [CrossRef]

39. Bredikhin, A.A.; Zakharychev, D.V.; Gubaidullin, A.T.; Fayzullin, R.R.; Samigullina, A.I.; Bredikhina, Z.A. Crystallization of chiral para- $n$-alkylphenyl glycerol ethers: Phase diversity and impressive predominance of homochiral guaifenesin-like supramolecular motif. Cryst. Grow. Des. 2018, 18, 3980-3987. [CrossRef]

40. Sheldrick, G.M. SHELXT-Integrated space-group and crystal-structure determination. Acta Crystallogr. Sect. A Found. Adv. 2015, 71, 3-8. [CrossRef] [PubMed]

41. Sheldrick, G.M. Crystal structure refinement with SHELXL. Acta Crystallogr. Sect. C Struct. Chem. 2015, 71, 3-8. [CrossRef] [PubMed]

42. Farrugia, L.J. WinGX and ORTEP for Windows: An update. J. Appl. Crystallogr. 2012, 45, 849-854. [CrossRef]

43. Macrae, C.F.; Edgington, P.R.; McCabe, P.; Pidcock, E.; Shields, G.P.; Taylor, R.; Towler, M.; van de Streek, J. Mercury: Visualization and analysis of crystal structures. J. Appl. Crystallogr. 2006, 39, 453-459. [CrossRef] 
44. DIFFRAC Plus Evaluation Package EVA, Version 11, User's Manual. Available online: https://www.bruker.com/content/bruker/ int/en/products-and-solutions / diffractometers-and-scattering-systems/x-ray-diffractometers/diffrac-suite-software / diffrac-eva.html (accessed on 23 March 2021).

45. Gubaidullin, A.T.; Samigullina, A.I.; Bredikhina, Z.A.; Bredikhin, A.A. Crystal structure of chiral ortho-alkyl phenyl ethers of glycerol: True racemic compound, normal, false and anomalous conglomerates within the single five-membered family. CrystEngComm 2014, 16, 6716-6729. [CrossRef]

46. Bredikhin, A.A.; Gubaidullin, A.T.; Bredikhina, Z.A.; Krivolapov, D.B.; Pashagin, A.V.; Litvinov, I.A. Absolute configuration and crystal packing for three chiral drugs prone to spontaneous resolution: Guaifenesin, methocarbamol and mephenesin. J. Mol. Struct. 2009, 920, 377-382. [CrossRef]

47. Jacques, J.; Collet, A.; Wilen, S.H. Enantiomers, Racemates, and Resolutions; Krieger Publishing Company: Malabar, FL, USA, 1994.

48. Meyerhoffer, W. Stereochemische Notizen. Ber. Dtsch. Chem. Ges. 1904, 37, 2604-2610. [CrossRef]

49. Bredikhin, A.A.; Bredikhina, Z.A. Stereoselective crystallization as a basis for single-enantiomer drug production. Chem. Eng. Technol. 2017, 40, 1211-1220. [CrossRef]

50. Castillo-Valles, M.; Martinez-Bueno, A.; Gimenez, R.; Sierra, T.; Ros, M.B. Beyond liquid crystals: New research trends for mesogenic molecules in liquids. J. Mater. Chem. C 2019, 7, 14454-14470. [CrossRef]

51. Tschierske, C. Mirror symmetry breaking in liquids and liquid crystals. Liq. Cryst. 2018, 45, 2221-2252. [CrossRef]

52. Lin, J.B.; Guo, Z.X.; Plas, J.; Amabilino, D.B.; De Feyter, S.; Schenning, A. Homochiral and heterochiral assembly preferences at different length scales-Conglomerates and racemates in the same assemblies. Chem. Commun. 2013, 49, 9320-9322. [CrossRef] [PubMed]

53. Corpinot, M.K.; Bucar, D.-K. A Practical guide to the design of molecular crystals. Cryst. Grow. Des. 2019, 19, 1426-1453. [CrossRef]

54. Jeffrey, G.A.; Saenger, W. Hydrogen Bonding in Biological Structures; Springer: Cham, Switzerland, 1994; 569p.

55. Bredikhin, A.A.; Bredikhina, Z.A.; Gubaidullin, A.T. Chirality-dependent supramolecular synthons based on the 1,3-oxazolidin2-one framework: Chiral drugs mephenoxalone, metaxalone and other 114 examples. CrystEngComm 2020, 22, $7252-7261$. [CrossRef] 